\title{
Looking through the lens of a sexual assault examiner: novel trends and approaches in forensic photography
}

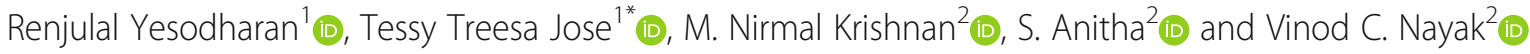

\begin{abstract}
Background: Sexual violence is cancerous, and it malignantly affects all strata of our community regardless of age, gender, race, ability, and social status. Excellent healthcare service requires professionals who can do a sexual assault examination, collect medico-legal evidence, take photographs of patients' anatomy that was involved in the assault and document what is there and where it is in relation to the victims or perpetrators body or other parts of the crime scene, whether or not it is connected to the crime.

Main text: The objective of this article to orient the professions to the novel trends and approaches in forensic photography, namely e-consent, digital cameras and their accessories, dedicated forensic software, and photographic protocols and procedures.

Conclusion: The inadequate and improper collection, preservation and presentation of evidence can lead to crime scene errors and fewer conviction rates. Training in forensic photography is vital and a game-changer in the sexual assault examination.
\end{abstract}

Keywords: SANE, SAFE, Forensic science, Forensic photography, Colposcopy, E-consent, Wound Photo Guide, Ring flashes

\section{Background}

Clinical photography could be medical or forensic; though they look similar, the aims are different (Henham and Lee 1994). The main purpose of medical photography is to illustrate a clinical finding, or an operative step or a postoperative result which aids in medical education and medical record keeping; whereas, clinical forensic photography is used to record and graphically document evidence for legal purposes because it is an element of legal proof (Bhattacharya 2014; Wittmann 2017). Forensic photography concerns with taking photographs of patients' anatomy (US Department of Justice 2013) that was involved in the assault and document what is there and where it is in relation to the victims or

\footnotetext{
*Correspondence: tessy.j@manipal.edu

'Department of Psychiatric Nursing, Manipal College of Nursing, Manipal

Academy of Higher Education, Manipal, Udupi, Karnataka, India

Full list of author information is available at the end of the article
}

perpetrators body or other parts of the crime scene, whether or not it is obviously connected to the crime. Sometimes these photographs can be used to show the progress of change in the injury or a wound or a comparison of findings in a follow-up visit (Edirisinghe et al. 2020) (US Department of Justice 2013). It also might be used for a second opinion. The images captured in the forensic examination might also be used in teaching and learning in medical and forensic education and professional development. Peer review and validation of document are essential factors which makes a forensic professional proficient in their profession. Whatever it may be, the precise role of these photographs is to eliminate bias. A single picture can potentially solve a case. It allows seeing things in places you cannot go. Remember, taking photos even if no obvious or visible signs of physical injury are vital in solving the case. As Wayne 
Gretzky wisely says, 'You will miss $100 \%$ of the shots that you fail to take'.

\section{Main text Informed consent}

Taking photographs even in normal circumstances can violate the integrity and privacy of the individuals; taking photographs after an assault on the examination table is traumatizing. Many patients may have undergone forensic examination, and it is not uncommon that they are frightened. Communication with the patient is essential in forensic examination, and the examiner should explain what is happening and what is going to happen next. Specially designed, informed consent informs the patient about risks, benefits, alternatives of medical and forensic evaluations, reporting the crime to law enforcing agencies, medico-legal examination, pregnancy testing, diagnosis and prophylaxis of sexually transmitted diseases (STDs), emergency contraception and photo documentation (Zerbo et al. 2018). It also includes the services and the limit of confidentiality (Yadav 2017). Informed consent (verbal and written) can help the patients understand the purpose of photography in the forensic examination and evidence collection (World Health Organization (WHO) 2003) (Ministry of Health and Family Welfare (MOHFW) 2014) (U.S. Department of Justice 2013). Patient consent must be taken before taking the photographs. The responsibility lies with the examiner to discuss the extent to which the photos will be taken and what procedures will be followed for preservation and storage. The information about the photos of the anogenital area and its use during the investigation and prosecution must be informed before the photography. The patient has the right to refuse all or any photography, and the refusal should be documented well in the medico-legal record as 'declined' rather than 'refused'. The forensic photographs taken should be kept in the forensic file and should not be kept along with the medical records to prevent unauthorized access (Thomas 2009).

\section{E-consent}

The widespread access to the evidence can be restricted by using an e-consent. The four forms of e-consents can be General consent, General consent with specific denial(s), General denial with specific consent(s), and General denial. E-consent permits access to confidential patient information only to those individuals who have been explicitly permitted by a patient and not to the one who have been denied access by the patient by invoking methods that check for explicit, inferred, or implied consent (Coiera and Clarke 2004).
General consent This type of consent uses an 'opt-in' model, which involves clear and affirmative actions. The client provides blanket consent for accessing any or all of their information by any health care professional working within a specified health context. This consent persists into the future unless specifically revoked by the patient.

General consent with specific denial(s) The clients provide general consent, but they exercised their right to restrict or deny certain aspects. These restrictions include disclosing particular information, disclosing to a specific party or category of parties, and disclosing for a particular purpose.

General denial with specific consent(s) The client denies all access to the information related to health but gives specific consent to the disclosure of particular information, disclosure to a particular party or parties or category of parties, and disclosure for a specific purpose.

General denial This type of denial uses an 'opt-out' model, and the client delivers a 'blanket denial' and denies consent for information to be used in future circumstances. The client is requested new consent to access the information each time.

The e-consent system can be used as a gatekeeper, where the system will permit only consented individuals to access information. The system also can prompt the individuals wishing to access a record that the client has expressed specific conditions about the use of their information and that a record of those conditions is available to read. The individual wishing to access information may then be asked to acknowledge that he or she understood the conditions before accessing the information (Coiera and Clarke 2004). The usage of econsents should not compromise human interactions. The privacy and confidentiality of the personal information are very critical, and the users accessing it should use proper authentication through either usernames and passwords, finger-drawn signatures, or biometrics (Chen et al. 2020).

\section{Comfort and privacy of the patient}

Comfort and privacy are at most important while conducting a forensic examination. People that do not need to be there in the examination room should not be there. Ensure privacy by pulling the curtains and lock the doors. Respect their need for modesty by pulling the drapes to the areas that are not being photographed. If the examiner wants to shift clothing to take a picture of the injury, place a blue sheet over the exposed area, making sure to only have the injury visible. A support person who the patient desires can be provided and 
necessary measures to avoid an allegation of impropriety should be considered. If the examiner is male, a female person should be present during the examination (Ministry of Health and Family Welfare (MOHFW) 2014). The examiner also needs to explain to them what he or she is going to do. Victim's protection is a paramount concern of any forensic team, and informing the incidents to any competent authorities or organizations should not cause an obstacle to the victim's protection (Tullio et al. 2021).

\section{Camera, accessories and software-selecting the right tools for the job \\ Digital camera}

A digital camera produces digital images stored in memory devices, displayed on-screen, and printed whenever required. When you consider a camera, it must have a removable storage medium, provision for the use of spare batteries, optical zoom lenses, better adjustment options, better light performances and provision for using synchronizing external accessories.

Single Lens Reflex (SLR) camera Digital SLR cameras are primarily used in forensic photography. The mirror in the DSLR camera directs the light from the lens allows the examiner to see what will be recorded once the photograph is taken. Using interchangeable lenses enables the examiner to have control over the composition, colour clarity, focus, speed, and depth of the field (Mancini and Sidoriak 2018). SLR camera can save the output in the RAW format, which has more colour details and sharpness than the JPEG format. The raw file is not an image format, but this can be converted to an image file. These formats have an advantage that it has more shades of colour, has finer control and adjustment potential, and does not suffer from image compressions. RAW images can be used as evidence of ownership and authenticity of the photograph.

Mirrorless camera These cameras do not have an intricate mirror system that makes them smaller, lighter, and simpler. The light goes through the sensor, gets processed and displayed in the camera monitor or electronic viewfinder. The modern-day mirrorless cameras have full-frame sensors and can compete with the digital SLR camera. This camera has limitations while taking pictures using manual focusing (Zoltie 2018) and shows lesser performance in the situation with low light.

Secure Digital Forensic Imaging (SDFI) camera system SDFI camera system includes a camera, memory cards, card reader, tripod, foot pedal, handheld remote and all the accessories to take forensic pictures. The standard camera system comes with autofocus and macro lens and can be used to take clear photos with an optimum view of the vaginal vault and the cervix. The autofocus lens helps the examiner to take sharp and clear images keeping the hands free. The recent update came is the SDFI-TeleMedicine Forensic Camera Systems (Secure Digital Forensic Imaging 2021).

SDFI contrast camera system It is designed with contrast lights to capture forensic images in the ultraviolet spectrum. The visible spectrum of light lies between 400 $\mathrm{nm}$ to $700 \mathrm{~nm}$, and contrast photography captures images in ultraviolet light and regular or low light. SDFI contrast camera and contrast light allow you to investigate, swab and photograph the crime scene, which includes the body of the victim. Dried fluids such as sperm and semen, and other things can be visualized in ultraviolet light, and the specially designed contrast camera can take pictures of these in dim or dark rooms (Secure Digital Forensic Imaging 2021).

Pocket Colposcope and Callascope Compared to a standard clinical Colposcope, the pocket colposcope can be inserted through the speculum to provide a close-up view of the cervix to take pictures with a 5-mega pixel camera. A Callascope is a tampon like introducer that is slender and allows a 2-mega pixel camera to take speculum-free images of the cervix (Asiedu et al. 2017) (Lam et al. 2015).

\section{Accessories}

Ring flash Regular flash may create shadows while taking photos of cavities of the body; a ring flash can solve this problem. These can be specifically used for taking pictures of the oral cavity (Ahmad 2009), vaginal cavity and anorectal regions (Fig. 1).

Tripod High-quality images need the camera to be in a good, still, fixed position to achieve the best. Tripod is needed to stabilize the camera. The examiner keeps moving while holding the camera can compromise the quality of the image. The tripods are made with aluminium alloy and carbon materials, and they come with three to five leg sections. A carbon fibre build keeps the tripod lightweight but strong. Tripods come with a ball head or three-way head. Three-way heads are larger but allow precise adjustments for each axis independently. (Richards 2021) A flexible mini tripod is a newer addition to this category.

Camera card Printing of photos is outdated. The recent trend is to store the images in removable storage devices, especially memory cards; examiners need to have multiple camera cards because using the same camera 


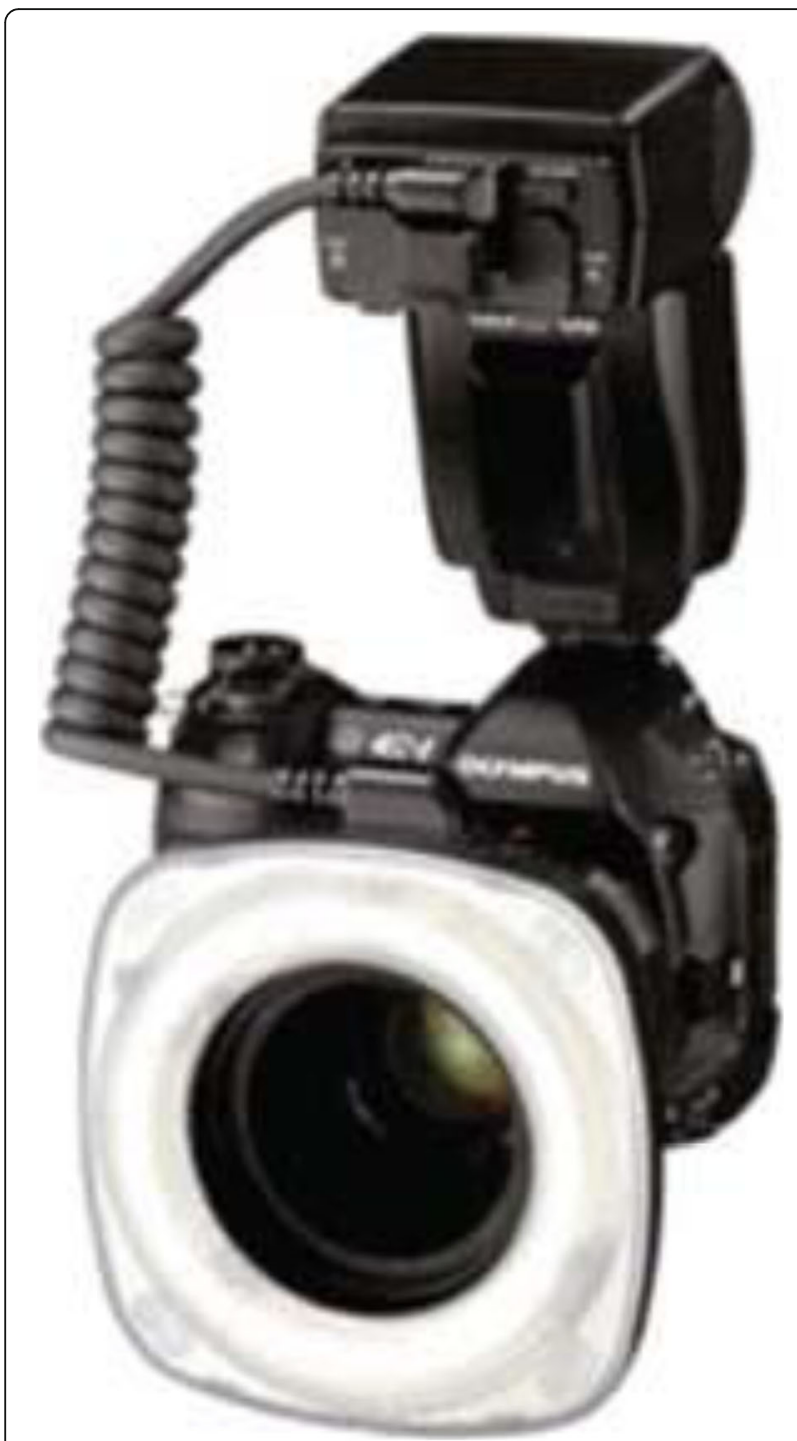

Fig. 1 The ring flash for the camera. Reprinted with permission from Ahmad I, General Dental Practitioner, The Ridgeway Dental Surgery, 173 The Ridgeway, North Harrow, Middlesex, HA2 7DF

card for different cases can cause mixing up of the photos. The use of photo ID cards can resolve this issue, but it is always better to use an empty camera card for every new case.

Battery Batteries are required for your camera as well as the flash. The camera and flash may require a lithiumion battery which is rechargeable or non-rechargeable Energizer batteries. If you are using rechargeable batteries, make sure that it is plugged in properly for charging and you need to have spare sets for unforeseen events. You may have to use a camera and flash multiple times a day and may need to get recharged in between. This may hamper the evidence collection. If you are using non-rechargeable Energizer batteries, make sure you have enough stock on supply.

Foot pedal/clicker The examiner sometimes needs both hands to tract the vaginal and anogenital area and wishes to take a photo in the same way. It will be nearly impossible to take such photos unless you have a pedaloperated clicker (Fig. 2).

Monitors Built-in Wi-Fi and Bluetooth connectivity in the modern-day cameras enable the examiners to see the camera view in the remote monitoring devices (including mobile phones) when the camera is fixed in the tripod, and the examiner's hands are not free. It helps the examiner see the visuals in the best suitable ankle without blocking the camera's view and operates a pedaloperated clicker to take photos. These monitoring devices also can be used to control the camera. Though these images captured can be shared using these devices and these can be misused, such devices need to be monitored as per the rules, regulations and laws of the state.

Camera filters Luminescence can be induced in fibres, biological fluids, pigments and inks, fingerprint development powders and petroleum products under the right conditions examined under light at specific wavelengths (Marin and Buszka 2013). UV light may allow the visualization of staining or pattern formations by the fluids if a coloured barrier filter may be used. This combination of coloured filter and UV light enhances the contrast of the subject matter for additional examinations (Lahm and Reitnauer 2015).

Blue or green backdrop The goal of the photography is to focus on the patient, the backgrounds which are untidy need to be consciously avoided (Nayler 2003). A plain neutral blue or green backdrop is essential to hide the minutia out of the picture. If a lady attendant is helping the person or a parent is present while doing the evidence collection, care should be taken to avoid including that person in the photograph.

Colouredgloves A coloured glow can be beneficial to clearly distinguish the body tissue while examining the Female Genitalia. The white glows become too light, which will have a poor focus on the tissue.

Coloured flags The examiner needs to take photos of dark-skinned or white-skinned individuals even when there is an absence of visual evidence of injury, but the patient may point out the site. The camera may not focus on the point where the injury is reported. Coloured flags placed on the reported area can help the 

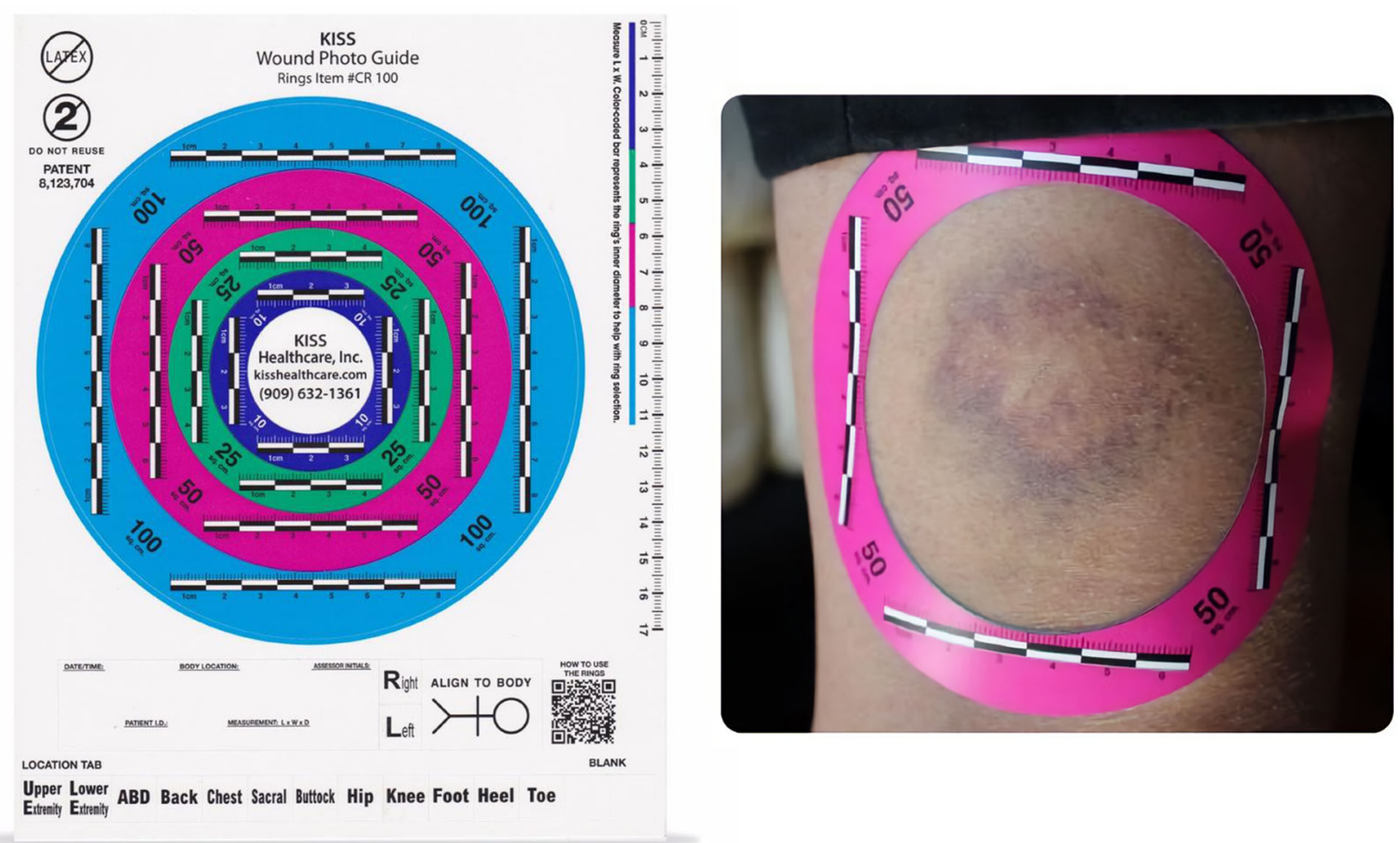

Fig. 2 The KISS wound photo guides_circle scales for back, chest, sacral, buttocks, hip, knee, foot, heel and toe (left). Application of the circle scale over an injured area for taking photography (Right). Reprinted with permission from TJ Richards, CEO, KISS Healthcare, Inc. KISS Wound address the problem of camera positioning by adheres to body's curvatures. The Rulers and L-rulers do not address these issues

camera to focus. These flags are coming in red, blue, yellow and green colours.

Photo ID card or photo identifiers These are used for taking orientation and bookend photography. It is the way to identify the pictures a particular examiner has taken. These identifiers will have options to write the date, name, age, gender, date of birth, case number, photographer name, and license number; they will be kept at the beginning and end of the series of photos taken by the examiner. The examiner can take a photo of the sticker or book card in the beginning and take photos of the patient and end the session by taking another photograph of the card again (Secure Digital Forensic Imaging 2020).

Hand map (right and left) It is a tool used for the hands, fingers, and fingernails' photo documentation process.

Scales, rulers, rings Measuring injuries are mandatory in sexual assault evidence collection, and they should be there in the photograph.

ABFO photomacrographic scales These are 'L' shaped rulers commonly used to demonstrate the size of the injury (Yesodharan et al. 2018). The long and short ' $\mathrm{L}$ ' rulers have a circle in the corner that can correct the focus while taking a photograph. These rulers are placed in a horizontal or vertical plane.

Adhesive scales These scales are 2" $(50 \mathrm{~mm})$ wide, they contain English and metric measurements printed on flat, pressure-sensitive label stock with a non-permanent adhesive back. These can be used on vertical or horizontal surfaces to measure fingerprints, traces of blood and bullet holes for photographic purposes.

The KISS wound photo guide-circle scales It is a patented photo scale design in rings and circles to reduce the subjectivity in wound photographs. This system is colour-coded to size and conforms to the curvature of the body. The rings are available in $18.7 \times 23.2 \mathrm{~cm} 1-9$, 10, 25, 50 and $100 \mathrm{sq}$. cm. Each ring has four linear scales to support the length and width of the wound in the photograph (Kiss Healthcare 2020) (Figs. 2 and 3).

Small adhesive numbers and arrow booklet The adhesive numbers and arrows are used to mark items in photographs. 
|ะแ|เก|

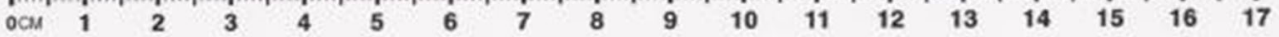

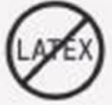

KISS

Wound Photo Guide

Rings Item \#CR1-9

INSTRUCTIONS ON THE BACK
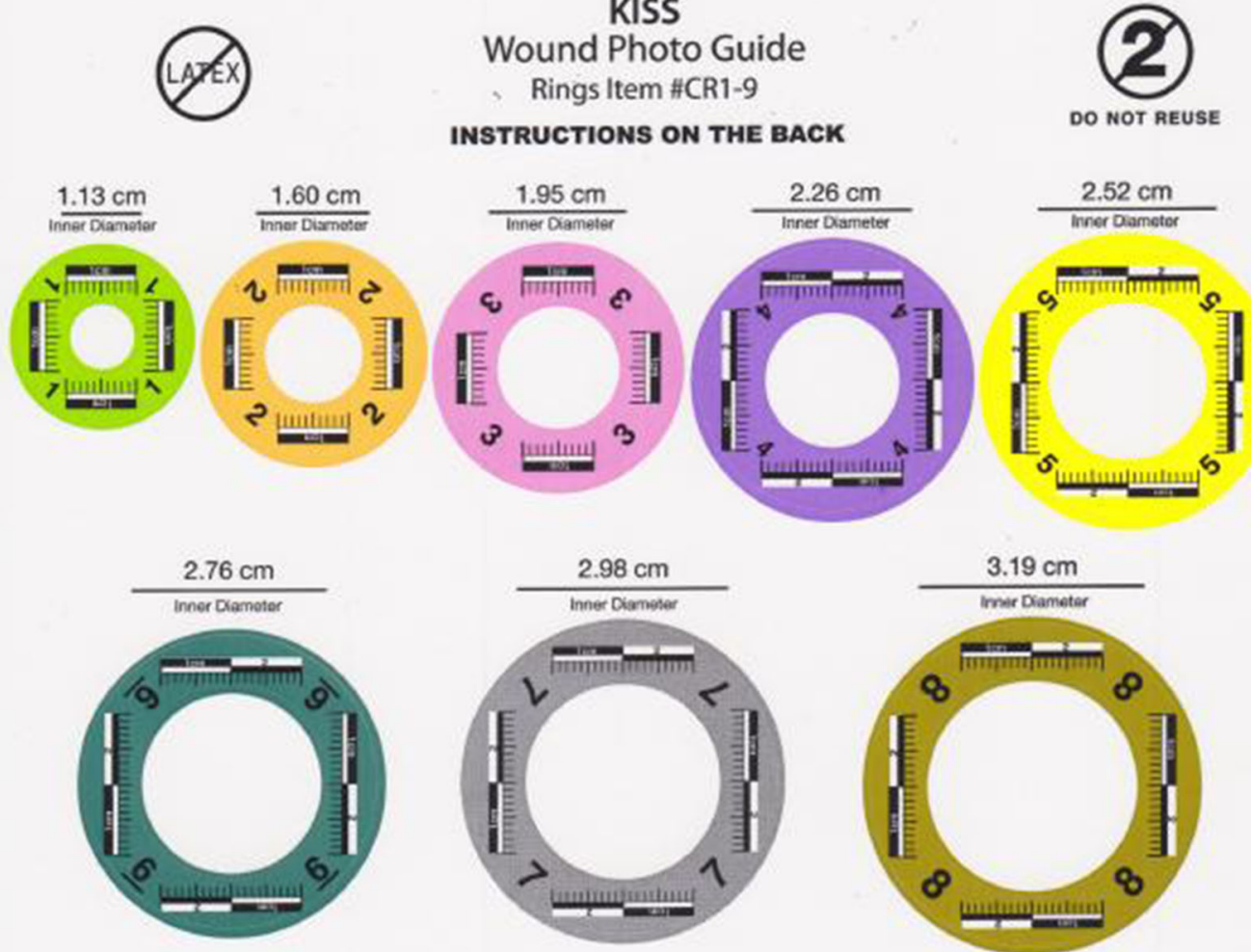

KISS HEALTHCARE, INC.

KISSHealthcare.com (909) 632-1361
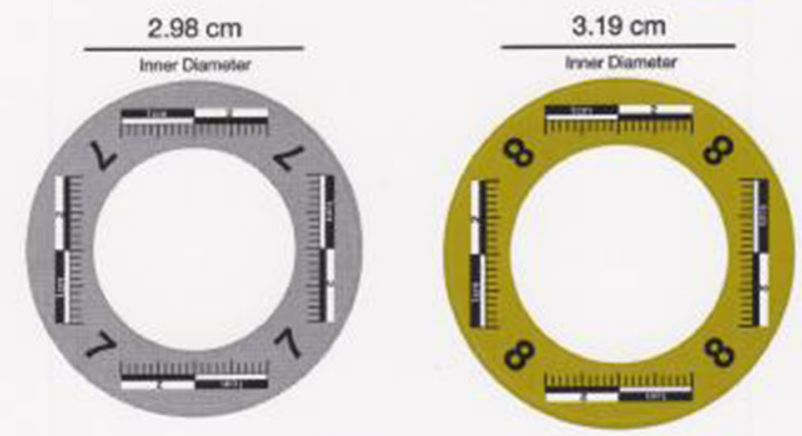

PATENT

$8,123,704$

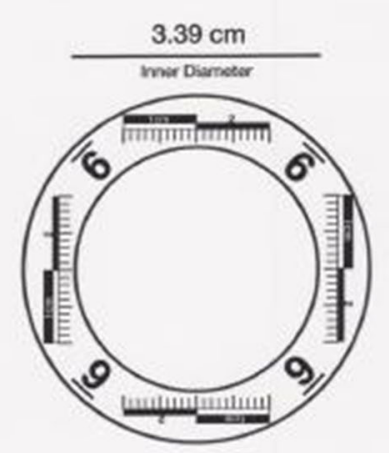

Fig. 3 The KISS wound photo guides_circle scales for knee, foot, heel and toe. Reprinted with permission from TJ Richards, CEO, KISS Healthcare, Inc.

\section{Dedicated forensic software's}

Image enhancement using digital filters (global contrast filters) Digital filters are scientifically accepted algorithms applied to the RBG values equally within the original image and make it easier to view its details. These filters are applied to the entire image and cause image enhancement by enhancing the pixel details. Image enhancement is not an image manipulation as the latter contains deletion, addition or alteration of any part of the image to create the desired look. When the manipulated data does not exist in the original image, it is not accepted as evidence in law courts. 


\section{Photography protocols, policies and procedures Orientation photography}

This includes taking eight to ten basic photographs, generally from the anterior, posterior, left and right sides of the body. The photos from each of these areas can be broken up into head to waist section and waist to feet section if the room size is small. Separate pictures of anterior and posterior aspects of the fingers of both hands also need to be taken. The orientation photos of the genital and the anal region need to be taken before the examination. Orientation photographs of genitals can be taken without and with the use of dye.

\section{Rule of $4 \mathrm{~s}$ and $5 \mathrm{~s}$}

The rule of $4 \mathrm{~s}$ include three different range of photography namely in distant range, mid-range and close up.

The distant photograph It should contain the injury and at least two identifiable landmarks.

The mid-range photograph It is closer than the distant photo by at least $50 \%$. The area of injury will be photographed so that it comes to the centre of the photo. Anatomical locations also can be included in this to avoid guessing.

The close-up photograph These photographs contain shots of injury at the centre of the frame. The photographer stands in front of the injury and takes photos straight. The first photo should be taken with a measuring scale or and then it can be removed and take a photo without the measuring scale.

In the rule of $5 \mathrm{~s}$, an additional closest image possible to capture the entire injury is taken. If the injury is large, overlapping images need to be taken from top-bottom, right and left. This creates a complete depiction of what is seen with naked eyes.

\section{Legal consideration}

The integrity of digital images can be assured. Some methods demonstrate digital file integrity, including hashing functions, visual verification, digital signatures, written documentation, and checksums/cyclical redundancy checks. Additionally, experts may determine whether a digital image, film photograph or film negative has been altered. When evidence is produced suggesting an alteration, experts can be used to confirm or refute the assertion.

\section{Conclusions}

Forensic experts and Sexual Assault Nurse Examiners should use technological advancements strictly in their practice. Capturing high-quality photos is one of these practices, comprising an important topic in forensic science.

\section{Abbreviations}

ABFO: American Board of Forensic Odontology; DSLR: Digital Single Lens Reflex; JPEG: Joint Photographic Experts Group; RBG: Red, Blue, Green; SDFI: Secure Digital Forensic Imaging; SLR: Single Lens Reflex

\section{Acknowledgements}

The authors acknowledge the contribution of TJ Richards, CEO, KISS Healthcare, Inc. and Ahmad, I, General Dental Practitioner, The Ridgeway Dental Surgery, 173 The Ridgeway, North Harrow, Middlesex, HA2 by providing images towards the article.

\section{Authors' contributions}

RY and NKM did a literature search in various databases and AS did a literature search in various educational websites, TTJ and VCN performed the evaluation of the articles and other literature, and all authors contributed to writing the manuscript. All authors read and approved the final manuscript.

\section{Funding}

The authors declared that there is no funding obtained for the preparation and publication of this article from any funding agency or institution from the public, commercial, or not-for-profit sectors.

Availability of data and materials

Not applicable.

\section{Declarations}

Ethics approval and consent to participate

This manuscript is a part of the study approved by Kasturba Medical College and Kasturba Hospital Institutional Ethics Committee through vide reference no $653 / 2018$. This manuscript does not report on or involve the use of any animal or human data or tissue.

Consent for publication

Not applicable.

\section{Competing interests}

All authors declare that they have no potential competing interest.

\section{Author details}

${ }^{1}$ Department of Psychiatric Nursing, Manipal College of Nursing, Manipal Academy of Higher Education, Manipal, Udupi, Karnataka, India. ${ }^{2}$ Department of Forensic Medicine and Toxicology, Kasturba Medical College Manipal, Manipal Academy of Higher Education, Manipal, Udupi, Karnataka, India.

Received: 4 May 2021 Accepted: 4 September 2021

Published online: 17 September 2021

\section{References}

Ahmad I (2009) Digital dental photography. Part 5: Lighting. Br Dent J 207(1):1318. https://doi.org/10.1038/sj.bdj.2009.558

Asiedu MN, Agudogo J, Krieger MS, Miros R, Proeschold-Bell RJ, Schmitt JW, Ramanujam N (2017) Design and preliminary analysis of a vaginal inserter for speculum-free cervical cancer screening. PLoS One 12(5):e0177782. https:// doi.org/10.1371/journal.pone.0177782

Bhattacharya S (2014) Clinical photography and our responsibilities. IndianJ Plast Surg 47(3):277-280. https://doi.org/10.4103/0970-0358.146569

Chen C et al (2020) Replacing Paper Informed Consent with Electronic Informed Consent for Research in Academic Medical Centers: A Scoping Review. In: AMIA Joint Summits on Translational Science proceedings. AMIA Joint Summits on Translational Science, pp 80-88 Available at: https://pubmed. ncbi.nlm.nih.gov/32477626

Coiera E, Clarke R (2004) e-Consent: The Design and Implementation of Consumer Consent Mechanisms in an Electronic Environment. J Am Med Inform Assoc 11(2):129-140. https://doi.org/10.1197/jamia.M1480

Edirisinghe PAS, Kitulwatte IDG, Nadeera DR (2020) Knowledge, attitude and practice regarding the use of digital photographs in the examination of the 
dead and living among doctors practicing forensic medicine in Sri Lanka. J Forensic Leg Med 73:101995. https://doi.org/10.1016/j.jflm.2020.101995

Henham AP, Lee KAP (1994) Photography in forensic medicine. J Vis Commun Med 17(1):15-20. https://doi.org/10.3109/17453059409018372

Kiss Healthcare (2020) The Kiss System- Wound Photo Scales. Available at: https:// kisssystem.com/+\&cd=1\&hl=en\&ct=clnk\&gl=in (Accessed: 21 Dec 2020).

Lahm C, Reitnauer A (2015) The Use of Colored Barrier Filters in Forensic Photography. Fingerprint Whorld 62(158) Available at: https://www.academia. edu/19475512/The_Use_of_Colored_Barrier_Filters_in_Forensic_Photography (Accessed: 7 Dec 2020)

Lam CT, Krieger MS, Gallagher JE, Asma B, Muasher LC, Schmitt JW, Ramanujam N (2015) Design of a Novel Low Cost Point of Care Tampon (POCkeT) Colposcope for Use in Resource Limited Settings. PLoS One. Edited by A. De 10(9):e0135869. https://doi.org/10.1371/journal.pone.0135869

Mancini K, Sidoriak J (2018) Fundamentals of forensic photography: practical techniques for evidence documentation on location and in the laboratory, 1st edn. Routledge Taylor \& Francis, New York

Marin N, Buszka MJ (2013) In: Miller SL (ed) Alternate Light Source Imaging: Forensic Photography Techniques, 1st edn. Anderson Publishing, New York. https://doi.org/10.4324/9781315722160

Ministry of Health and Family Welfare (MOHFW) (2014) Guidelines \& Protocols. Medico-legal care for survivors/victims of sexual violence. Available at: http:// mohfw.nic.in/sites/default/files/953522324.pdf (Accessed: 26 Aug 2017).

Nayler JR (2003) Clinical Photography : A Guide for the Clinician. J Postgrad Med 49(3):256-262

Richards, M. (2021) The best tripod in 2020 | Digital Camera World. Available at: https://www.digitalcameraworld.com/buying-guides/best-tripod (Accessed: 6 Dec 2020).

Secure Digital Forensic Imaging (2020) SDFI Bookend Cards Plus Hand Maps, Secure Digital Forensic Imaging. Available at: https://www.sdfi.com/ eCommerce/sdfi-forensic-photo-documentation-bookend-card (Accessed: 9 December 2020).

Secure Digital Forensic Imaging (2021) SDFI-TeleMedicine Forensic Photo Documentation System. Available at: https://www.sdfi.com/ (Accessed: 12 Mar 2021).

Thomas J (2009) Medical records and issues in negligence. Indian J Urol 25(3): 384-388. https://doi.org/10.4103/0970-1591.56208

Tullio V et al (2021) Violence against women in heterosexual couples: A review of psychological and medico-legal considerations. Med Sci Law 61(1_suppl): 113-124. https://doi.org/10.1177/0025802420936081

U.S. Department of Justice (2013) 'A National Protocol for Sexual Assault Medical Forensic Examinations - Adults/Adolescents'.

Wittmann A (2017) Overview of the Forensic Photography. J Forensic Sci Crim Investig 2(2):3-4. https://doi.org/10.19080/ffsci.2017.02.555581

World Health Organization (2003) Guidelines for medico-legal care of victims of sexual violence. World Health Organization, Geneva. https://apps.who.int/iris/ handle/10665/42788

Yadav PK (2017) Ethical issues across different fields of forensic science. Egypt J Forensic Sci 7(1):10. https://doi.org/10.1186/s41935-017-0010-1

Yesodharan R et al (2018) Forensic clinical photography: A game changer in medicolegal investigation and forensic science. Indian J Forensic Med Toxicol 12(2):262-266. https://doi.org/10.5958/0973-9130.2018.00113.5

Zerbo S, Milone L, Scalici E, Procaccianti S, Nardello R, Ventura Spagnolo E, Piscionieri D, Argo A (2018) Medico legal procedures related to sexual assault: a 10-year retrospective experience of a Daphne protocol application. Egypt J Forensic Sci 8(1):4. https://doi.org/10.1186/541935-018-0039-9

Zoltie T (2018) Mirrorless cameras for medical photography-time to switch? J Vis Commun Med 41(3):103-108. https://doi.org/10.1080/17453054.2018.1491938

\section{Publisher's Note}

Springer Nature remains neutral with regard to jurisdictional claims in published maps and institutional affiliations.

\section{Submit your manuscript to a SpringerOpen ${ }^{\circ}$ journal and benefit from:}

- Convenient online submission

- Rigorous peer review

- Open access: articles freely available online

- High visibility within the field

- Retaining the copyright to your article

Submit your next manuscript at $\boldsymbol{\nabla}$ springeropen.com 